\title{
Nonlinear Kato Class and Unique Continuation of Eigenfunctions for $p$-Laplacian Operator
}

\author{
René Erlín Castillo ${ }^{1}$ and Julio C. Ramos Fernández ${ }^{2}$ \\ ${ }^{1}$ Departamento de Matemáticas, Universidad Nacional de Colombia, Apartado, 360354 Bogotá, Colombia \\ ${ }^{2}$ Departamento de Matemáticas, Universidad de Oriente, Cumaná 6101 Estado Sucre, Venezuela
}

Correspondence should be addressed to René Erlín Castillo; recastillo@unal.edu.co

Received 25 April 2013; Revised 27 August 2013; Accepted 28 August 2013

Academic Editor: Janusz Matkowski

Copyright (C) 2013 R. E. Castillo and J. C. Ramos Fernández. This is an open access article distributed under the Creative Commons Attribution License, which permits unrestricted use, distribution, and reproduction in any medium, provided the original work is properly cited.

\begin{abstract}
We study some basic properties of nonlinear Kato class $M_{p}\left(\mathbb{R}^{n}\right)$ and $\widetilde{M}_{p}\left(\mathbb{R}^{n}\right)$, respectively, for $1<p<n$. Also, we study the problem $-\operatorname{div}\left(|\nabla u|^{p-2} \nabla u\right)+V|u|^{p-2} u=0$ in $\Omega$, where $\Omega$ is a bounded domain in $\mathbb{R}^{n}$ and the weight function $V$ is assumed to be not equivalent to zero and lies in $\widetilde{M}_{p}(\Omega)$, in the case where $p<n$. Finally, we establish the strong unique continuation property of the eigenfunction for the $p$-Laplacian operator in the case where $V \in \widetilde{M}_{p}(\Omega)$.
\end{abstract}

\section{Introduction}

The Kato class $K_{n}$ was introduced and studied by Aizenman and Simon (see [1]). For $n \geq 3$, it consists of locally integrable functions $f$ on $\mathbb{R}^{n}$, such that

$$
\lim _{r \rightarrow 0} \sup _{x \in \mathbb{R}^{n}} \int_{B(x, r)} \frac{|f(y)|}{|x-y|^{n-2}} d y=0 .
$$

For $1<p<n$, the following classes were defined by Zamboni (see [2]): the class $\widetilde{M}_{p}$ of functions $f$, such that

$$
\begin{aligned}
& \sup _{x \in \mathbb{R}^{n}}\left\{\int_{B(x, r)} \frac{1}{|x-y|^{n-1}}\left(\int_{B(x, r)} \frac{|f(z)|}{|y-z|^{n-1}} d z\right)^{1 /(p-1)} d y\right\}^{p-1} \\
& <\infty,
\end{aligned}
$$

and the class $M_{p}$ of functions $f$ such that $f \in \widetilde{M}_{p}$ and

$$
\begin{aligned}
\limsup _{r \rightarrow 0} \sup _{x \in \mathbb{R}^{n}}\left\{\int_{B(x, r)} \frac{1}{|x-y|^{n-1}}\right. \\
\left.\quad \times\left(\int_{B(x, r)} \frac{|f(z)|}{|y-z|^{n-1}} d z\right)^{1 /(p-1)} d y\right\}^{p-1}=0 .
\end{aligned}
$$

Section 2 of the present paper is devoted to the study of some basic properties of the nonlinear Kato class $M_{p}\left(\mathbb{R}^{n}\right)$ and $\widetilde{M}_{p}\left(\mathbb{R}^{n}\right)$, respectively, for $1<p<n$.

Among other things, we show that the Lorentz space $L(n / 2,1)$ is embedded into $M_{p}\left(\mathbb{R}^{n}\right)$ (see Lemma 10) as well as that $\widetilde{M}_{p}\left(\mathbb{R}^{n}\right)$ is a complete topological vector space (see Remark 11 and Lemma 13).

The $p$-Laplacian operator is a generalization of the Laplace operator, where $p$ is allowed to range over $1<p<\infty$; 
in our case where $1<p<n$, it is written as

$$
\begin{aligned}
\operatorname{div} & \left(|\nabla u|^{p-2} \nabla u\right) \\
& =\nabla \cdot\left(|\nabla u|^{p-2} \nabla u\right) \\
& =|\nabla u|^{p-4}\left(|\nabla u|^{2} \Delta u+(p-2) \sum_{i, j=1}^{n} \frac{\partial u}{\partial x_{i}} \frac{\partial u}{\partial x_{j}} \frac{\partial^{2} u}{\partial x_{i} \partial x_{j}}\right),
\end{aligned}
$$

where

$$
\nabla u=\left(\frac{\partial u}{\partial x_{1}}, \ldots, \frac{\partial u}{\partial x_{n}}\right), \quad|\nabla u|=\left(\sum_{i=1}^{n}\left(\frac{\partial u}{\partial x_{i}}\right)^{2}\right)^{1 / 2}
$$

and $u \in C_{0}^{\infty}(\Omega)$, with $\Omega$ bounded domain in $\mathbb{R}^{n}$.

We are concerned with the following problem:

$$
-\operatorname{div}\left(|\nabla u|^{p-2} \nabla u\right)+V|u|^{p-2} u=0, \quad \text { in } \Omega,
$$

and the weight function $V$ is assumed to be not equivalent to zero and lies in $\widetilde{M}_{p}\left(\mathbb{R}^{n}\right)$ in the case $p<n$.

Specifically, we are interested in studying a family of functions which enjoys the strong unique continuation property, that is, functions besides the possible zero functions which have zero of infinite order.

Definition 1. We say that a function $u \in L_{\text {loc }}^{p}(\Omega)$ vanishes of infinite order at point $x_{0}$ if for any natural number $N$ there exists a constant $C_{N}$, such that

$$
\int_{B\left(x_{0}, r\right)}|u(x)|^{p} d x \leq C_{N} r^{N}
$$

for all $N \in \mathbb{N}$ and for small positive number $r$. Here,

$$
B\left(x_{0}, r\right)=\left\{y \in \mathbb{R}^{n}:\left|y-x_{0}\right|<r\right\} .
$$

Definition 2. We say that (6) has a strong unique continuation property if and only if any solution $u$ of (6) in $\Omega$ is identically zero in $\Omega$ provided that $u$ vanishes of infinite order at a point in $\Omega$.

There is an extensive literature on unique continuation. We refer to the work of Zamboni on unique continuation for nonnegative solutions of quasilinear elliptic equation [3], also the work of Jerison-Kenig on the unique continuation for Schrödinger operators [4]. The same work is done by Chiarenza and Frasca, but for linear elliptic operator in the case where $V \in L^{n / 2}$ when $n>2$ [5].

Let us recall some known results concerning Fefferman's inequality as follows:

$$
\int_{\mathbb{R}^{n}}|u(x)|^{p}|V(x)| d x \leq C \int_{\mathbb{R}^{n}}|\nabla u(x)|^{p} d x \quad \forall u \in C_{0}^{\infty}\left(\mathbb{R}^{n}\right) .
$$

In [6], de Figueiredo and Gossez prove (9) in the case where $p=2$, assuming that $V \in L^{r, n-2 r}\left(\mathbb{R}^{n}\right)$ with $1<r \leq n / 2$. Later in [7], Jerison and Kening showed the same result taking $V$ in the Stummel-Kato class $S\left(\mathbb{R}^{n}\right)$. We point out that it not possible to compare the assumptions $V \in L^{r, n-2 r}\left(\mathbb{R}^{n}\right)$ and $f \in S\left(\mathbb{R}^{n}\right)$. Chiarenza and Frasca [5] generalized Fefferman's result proving (9) under the assumption that $V \in L^{r, n-2 r}\left(\mathbb{R}^{n}\right)$ with $r \in(1, n / p)$ and $p \in(1, n)$. In [3], Schechter gave a new proof of (9) assuming that $V \in \widetilde{M}_{p}\left(\mathbb{R}^{n}\right)$.

\section{Definitions and Notation}

In this section, we gather definitions and notations that will be used throughout the paper. We also include several simple lemmas. By $L_{\text {loc }}^{1}\left(\mathbb{R}^{n}\right)$, we will denote the space of functions which are locally integrable on $\mathbb{R}^{n}$, and by $L_{\text {loc }, u}^{1}$ the space of functions $f$, such that

$$
\sup _{x \in \mathbb{R}^{n}} \int_{B(x, 1)}|f(y)| d y<\infty .
$$

Definition 3. Let $f \in L_{\text {loc }}^{1}\left(\mathbb{R}^{n}\right)$. For any $1<p<n$ and $r>0$, we set

$\Phi(r)$

$$
=\sup _{x \in \mathbb{R}^{n}}\left(\int_{B(x, r)} \frac{1}{|x-y|^{n-1}}\left(\int_{B(x, r)} \frac{|f(z)| d z}{|z-y|^{n-1}}\right)^{1 /(p-1)} d y\right)^{p-1},
$$

where $B(x, r)=\{y:|x-y|<r\}$. We say that $f$ belongs to the space $\widetilde{M}_{p}\left(\mathbb{R}^{n}\right)$ if $\Phi(r)<\infty$ for all $r>0$.

Definition 4. We say that a function $f \in M_{p}\left(\mathbb{R}^{n}\right)$ if

$$
\lim _{r \rightarrow 0} \Phi(r)=0
$$

We are now ready to formulate some simple properties of the classes $M_{p}$ and $\widetilde{M}_{p}$.

Lemma 5 (see [3], page 152). For $1<p<n$, one has

(i) $M_{p}\left(\mathbb{R}^{n}\right) \subset \widetilde{M}_{p}\left(\mathbb{R}^{n}\right)$,

(ii) $M_{2}\left(\mathbb{R}^{n}\right)=K_{n}$.

From Lemma 5 we conclude that both $M_{p}\left(\mathbb{R}^{n}\right)$ and $\widetilde{M}_{p}\left(\mathbb{R}^{n}\right)$ are generalizations of $K_{n}$.

Remark 6. The following example shows that $K_{n}$ is properly contained in $M_{p}\left(\mathbb{R}^{n}\right)$ for $p>2$. It is known that the function 
$f(x)=|x|^{-2}$ is not in the Kato class $K_{n}$. However, $f \in M_{p}$. Indeed,

$$
\begin{aligned}
\lim _{r \rightarrow 0} \sup _{x}\left\{\int_{B(x, r)} \frac{1}{|x-y|^{n-2}}\right. \\
\left.\quad \times\left(\int_{B(x, r)} \frac{d z}{|z|^{2}|z-y|^{n-1}}\right)^{1 /(p-1)} d y\right\}^{p-1}=0 .
\end{aligned}
$$

This can be shown by splitting the domain of integration in the interior integral into the following three parts: $B(x, r) \bigcap\{|z|<(1 / 2)|y|\}, B(x, r) \bigcap\{(1 / 2)|y| \leq|z| \leq$ $(3 / 2)|y|\}$, and $B(x, r) \bigcap\{|z|>(3 / 2)|y|\}$.

After routine calculations, we can see that

$$
\int_{B(x, r)} \frac{d z}{|z|^{2}|z-y|^{n-1}}
$$

is majorized by $C|y|^{-1}$. Finally, we have

$$
C \sup _{x}\left\{\int_{B(x, r)} \frac{d y}{|y|^{1 /(p-1)}|x-y|^{n-1}}\right\}^{p-1} \rightarrow 0 \quad \text { as } r \longrightarrow 0
$$

This shows that (13) holds. Thus, $f \in \bigcap_{p>2} M_{p}$.

Definition 7. The distribution function $D_{f}$ of a measurable function $f$ is given by

$$
D_{f}(\lambda)=m\left(\left\{x \in \mathbb{R}^{n}:|f(x)|>\lambda\right\}\right),
$$

where $m$ denotes the Lebesgue measure on $\mathbb{R}^{n}$. The distribution function $D_{f}$ provides information about the size of $f$ but not about the behavior of $f$ itself near any given point. For instance, a function on $\mathbb{R}^{n}$ and each of its translates have the same distribution function. It follows from Definition 7 that $D_{f}$ is a decreasing function of $\lambda$ (not strictly necessary).

Definition 8. Let $f$ be a measurable function in $\mathbb{R}^{n}$. The decreasing rearrangement of $f$ is the function $f$ defined on $[0, \infty)$ by

$$
f^{*}(t)=\inf \left\{\lambda: D_{f}(\lambda) \leq t\right\} \quad(t \geq 0) .
$$

We use here the convention that inf $\emptyset=\infty$.

Definition 9 (Lorentz space). Let $f$ be a measurable function; we say that $f$ belongs to $L_{(n / 2,1)}$ if

$$
\|f\|_{(n / 2,1)}=\int_{0}^{\infty} t^{2 / n-1} f^{*}(t) d t<\infty .
$$

And it belongs to $L(n /(n-2), \infty)$ if

$$
\|f\|_{(n /(n-2), \infty)}=\sup _{t>1} t^{1-2 / n} f^{*}(t)<\infty .
$$

Lemma 10. Consider $L_{(n / 2,1)} \subset M_{p}\left(\mathbb{R}^{n}\right)$.

Proof. Let $f \in L_{(n / 2,1)}$; then

$$
\int_{0}^{\infty} t^{2 / n-1} f^{*}(t) d t<\infty .
$$

Since $|f| \chi_{B(x, r)} \leq|f|$, we have

$$
\left(|f| \chi_{B(x, r)}\right)^{*}(t) \leq f^{*}(t)
$$

then,

$$
\int_{0}^{\infty} t^{2 / n-1}\left(|f| \chi_{B(x, r)}\right)^{*}(t) d t \leq \int_{0}^{\infty} t^{2 / n-1} f^{*}(t) d t<\infty .
$$

Thus, $|f| \chi_{B(x, r)} \in L_{(n / 2,1)}$.

On the other hand, let $g(x)=|x|^{-(1-n)}$; then

$$
\begin{aligned}
& m(\{x:|g(x)|>\lambda\})=m\left(\left\{x:|x|^{-(1-n)}>\lambda\right\}\right), \\
& m\left(\left\{x:|x|<\left(\frac{1}{\lambda}\right)^{1 /(n-1)}\right\}\right)=C_{n}\left(\frac{1}{\lambda}\right)^{n /(n-1)},
\end{aligned}
$$

where $C_{n}=m(B(0,1))$.

Next, we set $t=C_{n}(1 / \lambda)^{n /(n-1)}$, and then $\lambda=C_{n} t^{1 / n-1}$. Thus, $g^{*}(t)=C_{n} t^{1 / n-1}$. From this, we obtain

$$
\begin{aligned}
& \|g\|_{(n /(n-2), \infty)} \\
& \quad=\left\|\frac{1}{|\cdot|^{n-1}}\right\|_{(n /(n-2), \infty)} \\
& \quad=\sup _{t>1} C_{n} t^{1-2 / n} t^{1 / n-1}=\sup _{t>1} C_{n} t^{-(1 / n)} \\
& \quad \leq C_{n}<\infty,
\end{aligned}
$$

which means that $g \in L_{(n /(n-2), \infty)}$. Finally, by Fubini's theorem and Hölder's inequality, we have

$\phi(r)$

$$
\begin{aligned}
& =\sup _{x \in \mathbb{R}^{n}}\left(\int_{B(x, r)} \frac{1}{|x-y|^{n-1}}\right. \\
& \left.\quad \times\left(\int_{B(x, r)} \frac{|f(z)|}{|y-z|^{n-1}} d z\right)^{1 /(p-1)} d y\right)^{p-1} \\
& \leq \sup _{x \in \mathbb{R}^{n}}\left(\int_{B(x, r)} \frac{1}{|x-y|^{n-1}}\right. \\
& \left.\times\left(\int_{B(x, 2 r)} \frac{|f(z)|}{|y-z|^{n-1}} d z\right)^{1 /(p-1)} d y\right)^{p-1} \\
& =\sup _{x \in \mathbb{R}^{n}}\left(\int_{B(x, r)} \frac{1}{|x-y|^{n-1}}\right.
\end{aligned}
$$

$$
\left.\times\left(\int_{\mathbb{R}^{n}} \frac{|f(z)| \chi_{B(0,2 r)}(y-z) d z}{|y-z|^{n-1}}\right)^{1 /(p-1)} d y\right)^{p-1}
$$




$$
\begin{aligned}
& \leq \sup _{x \in \mathbb{R}^{n}}\left(\int_{B(x, r)} \frac{d y}{|x-y|^{n-1}}\right)^{p-1} \\
& \quad \times\left\|f \chi_{B(0,2 r)}\right\|_{(n / 2,1)}\left\|\frac{1}{|\cdot|^{n-1}}\right\|_{(n /(n-2), \infty)} \\
&=C_{n} r^{p-1}\left\|f \chi_{B(0,2 r)}\right\|_{(n / 2,1)} \longrightarrow 0 \quad \text { as } r \longrightarrow 0,
\end{aligned}
$$

which means that $f \in M_{p}\left(\mathbb{R}^{n}\right)$ and the proof is complete.

Remark 11. (i) For $0<r<1$, it is not hard to check that for $1<p \leq 2$, the expression

$$
\begin{aligned}
& \|f\|_{\widetilde{M}_{p}\left(\mathbb{R}^{n}\right)} \\
& =\sup _{x \in \mathbb{R}^{n}}\left(\int_{B(x, 1)} \frac{1}{|x-y|^{n-1}}\right. \\
& \left.\quad \times\left(\int_{B(x, 1)} \frac{|f(z)|}{|z-y|^{n-1}} d z\right)^{1 /(p-1)} d y\right)^{p-1}
\end{aligned}
$$

defines a norm on $\widetilde{M}_{p}\left(\mathbb{R}^{n}\right)$.

(ii) For $p>2$, the expression (26) satisfies the following inequality:

$$
\|f+g\|_{\widetilde{M}_{p}\left(\mathbb{R}^{n}\right)} \leq 2^{p-2}\left(\|f\|_{\widetilde{M}_{p}\left(\mathbb{R}^{n}\right)}+\|g\|_{\widetilde{M}_{p}\left(\mathbb{R}^{n}\right)}\right)
$$

for all $f$ and $g$ in $\widetilde{M}_{p}\left(\mathbb{R}^{n}\right)$.

If $U$ is a neighborhood of 0 from (27), we have

$$
2^{p-1} U+2^{p-1} U \subset U
$$

then, $\widetilde{M}_{p}\left(\mathbb{R}^{n}\right)$ is a topological vector space.

Lemma 12. Consider $\widetilde{M}_{p}\left(\mathbb{R}^{n}\right) \subset L_{\text {loc }, u}^{1}\left(\mathbb{R}^{n}\right)$ for $1<p<n$.

Proof. Let $f \in \widetilde{M}_{p}\left(\mathbb{R}^{n}\right)$, and fix $r_{0}>0$. Then, there exists a positive constant $C$ such that $\Phi\left(r_{0}\right) \leq C$. It follows that

$$
\begin{gathered}
\sup _{x \in \mathbb{R}^{n}}\left(\int_{B\left(x, r_{0}\right)} \frac{1}{|x-y|^{n-1}}\left(\int_{B\left(x, r_{0}\right)} \frac{|f(z)|}{|z-y|^{n-1}} d z\right)^{1 /(p-1)} d y\right)^{p-1} \\
\geq \sup _{x \in \mathbb{R}^{n}}\left(\int_{B\left(x, r_{0}\right)} \frac{d y}{r_{0}^{n-1}}\left(\int_{B\left(x, r_{0}\right)} \frac{|f(z)|}{\left(2 r_{0}\right)^{n-1}} d z\right)^{1 /(p-1)}\right)^{p-1} \\
\geq \sup _{x \in \mathbb{R}^{n}}\left(\frac{1}{2 r_{0}}\right)^{n-1}\left(\frac{m\left(B\left(x, r_{0}\right)\right)}{r_{0}^{n-1}}\right)^{p-1} \int_{B\left(x, r_{0}\right)}|f(z)| d z .
\end{gathered}
$$

Therefore,

$$
\sup _{x \in \mathbb{R}^{n}} \int_{B\left(x, r_{0}\right)}|f(z)| d z<B C
$$

where

$$
B=\left(2 r_{0}\right)^{n-1}\left(r_{0} m(B(0,1))\right)^{p-1} .
$$

Finally, let $B(x, 1) \subseteq \bigcup_{k=1}^{n} B\left(x_{k}, r_{0}\right)$; then

$$
\sup _{x \in \mathbb{R}^{n}} \int_{B(x, 1)}|f(z)| d z \leq \sum_{k=1}^{n} \sup _{x \in \mathbb{R}^{n}} \int_{B\left(x_{k}, r_{0}\right)}|f(z)| d z,
$$

so

$$
\sup _{x \in \mathbb{R}^{n}} \int_{B(x, 1)}|f(z)| d z<\infty .
$$

Therefore,

$$
\widetilde{M}_{p}\left(\mathbb{R}^{n}\right) \subset L_{\text {loc }, u}^{1}\left(\mathbb{R}^{n}\right)
$$

Lemma 13. For $1<p<n, \widetilde{M}_{p}\left(\mathbb{R}^{n}\right)$ is a complete space.

Proof. Let $\left\{f_{n}\right\}_{n \in \mathcal{N}}$ be a Cauchy sequence in

$$
\bar{B}(0, r)=\left\{f \in \widetilde{M}_{p}\left(\mathbb{R}^{n}\right):\|f\|_{\widetilde{M}_{p}\left(\mathbb{R}^{n}\right)} \leq r\right\} .
$$

By Lemma 10, $\left\{f_{n}\right\}_{n \in \mathcal{N}}$ is a Cauchy sequence in $L_{\text {loc }, u}^{1}\left(\mathbb{R}^{n}\right)$. Since this space is complete, there exists a function $f \in$ $L_{\text {loc }, u}^{1}\left(\mathbb{R}^{n}\right)$ such that $f_{n} \rightarrow f$ in $L_{\text {loc }, u}^{1}\left(\mathbb{R}^{n}\right)$. By Fatous's lemma, we have

$$
\|f\|_{\widetilde{M}_{p}\left(\mathbb{R}^{n}\right)} \leq \liminf \left\|f_{n}\right\|_{\widetilde{M}_{p}\left(\mathbb{R}^{n}\right)} \leq r .
$$

Thus, $f \in \bar{B}(0, r)$, which means that $\bar{B}(0, r)$ is complete with respect to the topology generated by $L_{\text {loc,u }}^{1}\left(\mathbb{R}^{n}\right)$-norm. By Corollary 2 of Proposition 9 in [8, Chapter III, Section 3, no. 5] we obtain the assertion.

Lemma 14. If $1<p<n$, then $M_{p}\left(\mathbb{R}^{n}\right)$ is closed in $\widetilde{M}_{p}\left(\mathbb{R}^{n}\right)$.

Proof. Let us define the map $\varphi: \widetilde{M}_{p}\left(\mathbb{R}^{n}\right) \rightarrow[0, \infty)$ by $\varphi(f)=\lim _{r \rightarrow 0} \phi_{f}(r)$ (see Definition 3 ).

It is not hard to prove that the family $\left\{\varphi_{r}\right\}_{r>0}$ where $\varphi_{r}(f)=\phi_{f}(r)$ is equicontinuous and $\varphi_{r} \rightarrow \varphi$ pointwise as $r \rightarrow 0$. Since $M_{p}\left(\mathbb{R}^{n}\right)=\varphi^{-1}(0)$, we obtain the result.

For more details on nonlinear Kato class, we refer the readers to [9].

\section{Some Useful Inequalities}

For the sake of completeness and convenience of the reader, we include the proof of the next result which is due to Schechter [3].

Theorem 15. Assume that $V \in \widetilde{M}_{p}\left(\mathbb{R}^{n}\right)$. Then, for any $r>0$ there exists a positive constant $C(n, p)$, such that

$$
\int_{\mathbb{R}^{n}}|V(x)||u(x)|^{p} d x \leq C(n, p) \Phi(2 r) \int_{\mathbb{R}^{n}}|\nabla u(x)|^{p} d x,
$$

for any $u \in C_{0}^{\infty}\left(\mathbb{R}^{n}\right)$ supported in $B\left(x_{0}, r\right)$. 
Proof. For any $u \in C_{0}^{\infty}\left(\mathbb{R}^{n}\right)$ supported in $B\left(x_{0}, r\right)$, using the well-known inequality

$$
|u(x)| \leq C(n, p) \int_{B\left(x_{0}, r\right)} \frac{|\nabla u(y)|}{|x-y|^{n-1}} d y,
$$

Fubini's theorem, and Hölder's inequality, we obtain

$$
\begin{aligned}
& \int_{\mathbb{R}^{n}}|V(x)||u(x)|^{p} d x \\
& =\int_{B\left(x_{0}, r\right)}|V(x)||u(x)|^{p} d x \\
& \leq C(n, p) \int_{B\left(x_{0}, r\right)}|V(x)||u(x)|^{p-1} \\
& \times\left(\int_{B\left(x_{0}, r\right)} \frac{|\nabla u(y)|}{|x-y|^{n-1}} d y\right) d x \\
& \leq C(n, p) \int_{B\left(x_{0}, r\right)}|\nabla u(y)| \\
& \times\left(\int_{B\left(x_{0}, r\right)}|V(x)||u(x)|^{p-1} \frac{1}{|x-y|^{n-1}} d x\right) d y \\
& \leq C(n, p)\left(\int_{B\left(x_{0}, r\right)}|\nabla u(y)|^{p} d y\right)^{1 / p} \\
& \times\left[\int _ { B ( x _ { 0 } , r ) } \left(\int_{B\left(x_{0}, r\right)}|V(x)||u(x)|^{p-1}\right.\right. \\
& \left.\left.\times \frac{1}{|x-y|^{n-1}} d x\right)^{p /(p-1)} d y\right]
\end{aligned}
$$

On the other hand, using Hölder's inequality one more time, we have

$$
\begin{aligned}
& \int_{B\left(x_{0}, r\right)}\left(\int_{B\left(x_{0}, r\right)}|V(x)||u(x)|^{p-1} \frac{1}{|x-y|^{n-1}} d x\right)^{p /(p-1)} d y \\
& \leq \int_{B\left(x_{0}, r\right)}\left(\int_{B\left(x_{0}, r\right)} \frac{|V(z)|}{|z-y|^{n-1}} d z\right)^{1 /(p-1)} \\
& \quad \times \int_{B\left(x_{0}, r\right)} \frac{|V(x)||u(x)|^{p}}{|x-y|^{n-1}} d x d y \\
& =\int_{B\left(x_{0}, r\right)}|V(x)||u(x)|^{p} \\
& \quad \times \int_{B\left(x_{0}, r\right)} \frac{1}{|x-y|^{n-1}}\left(\int_{B\left(x_{0}, r\right)} \frac{|V(z)|}{|z-y|^{n-1}} d z\right)^{1 /(p-1)} d y d x \\
& \leq[\Phi(2 r)]^{1 /(p-1)} \int_{B\left(x_{0}, r\right)}^{|V(x)||u(x)|^{p} d x .}
\end{aligned}
$$

By (39) and (40), we obtain

$$
\begin{aligned}
& \int_{B\left(x_{0}, r\right)}|V(x)||u(x)|^{p} d x \\
& \leq C(n, p)[\Phi(2 r)]^{1 /(p-1)}\left(\int_{B\left(x_{0}, r\right)}|\nabla u(y)|^{p} d y\right)^{1 / p} \\
& \quad \times\left(\int_{B\left(x_{0}, r\right)}|V(x)||u(x)|^{p} d x\right)^{1-1 / p} .
\end{aligned}
$$

Hence

$$
\begin{aligned}
\int_{B\left(x_{0}, r\right)} & |V(x)||u(x)|^{p} d x \\
\leq & C(n, p)[\Phi(2 r)]^{q / p} \int_{B\left(x_{0}, r\right)}|\nabla u(x)|^{p} d x .
\end{aligned}
$$

The next corollary is an easy consequence of the previous theorem. It can be obtained via a standard partition of unity.

Corollary 16. Let $V \in \widetilde{M}_{p}\left(\mathbb{R}^{n}\right)$ and let $\Omega$ be a bounded subset of $\mathbb{R}^{n}$, supp $V \subseteq \Omega$. Then, for any $\sigma>0$ there exists a positive constant $K$ depending on $\sigma$, such that

$$
\begin{aligned}
& \int_{\Omega}|V(x)||u(x)|^{p} d x \\
& \quad \leq \sigma \int_{\Omega}|\nabla u(x)|^{p} d x+K(\sigma) \int_{\Omega}|u(x)|^{p} d x,
\end{aligned}
$$

for all $u \in C_{0}^{\infty}(\Omega)$

Proof. Let $\sigma>0$. Let $r$ be a positive number that will be chosen later. Let $\left\{\alpha_{k}^{p}\right\}, k=1,2, \ldots, N(r)$, be a finite partition of the unity of $\bar{\Omega}$, such that $\operatorname{supp} V \subseteq B\left(x_{k}, r\right)$ with $x_{k} \in \bar{\Omega}$. We apply Theorem 15 to the functions $\alpha_{k}$ and we get

$$
\begin{aligned}
\int_{\Omega} & |V(x)||u(x)|^{p} d x \\
& =\int_{\Omega}|V(x)||u(x)|^{p} \sum_{k=1}^{N(r)} \alpha_{k}^{p}(x) d x \\
& =\sum_{k=1}^{N(r)} \int_{\Omega}|V(x)|\left|u(x) \alpha_{k}^{p}(x)\right|^{p} d x \\
\leq & \sum_{k=1}^{N(r)} C \Phi_{V}(2 r)\left(\int_{\Omega}|\nabla u(x)|^{p} \alpha_{n}^{p}(x) d x\right. \\
\leq & C \Phi_{V}(2 r)\left(\int_{\Omega}|\nabla u(x)|^{p} d x+\frac{N(r)}{r^{p}} \int_{\Omega}|u(x)|^{p} d x\right) .
\end{aligned}
$$

Finally, to obtain the result, it is sufficient to choose $r$ such that $C \Phi_{V}(2 r)=\sigma$. After that, we note that $N(r) \approx r^{-n}$ and the corollary follows. 
Lemma 17. Let $B_{r}$ and $B_{2 r}$ be two concentric balls contained in $\Omega$. Then,

$$
\int_{B_{r}}|\nabla u(x)|^{p} d x \leq \frac{C}{r^{p}} \int_{B_{2 r}}|u|^{p},
$$

where the constant $C$ does not depend on $r$ and $u \in C_{0}^{\infty}\left(B_{r}\right)$.

Proof. Take $\varphi \in C_{0}^{\infty}(\Omega)$, with supp $\varphi \subset B_{2 r}, \varphi(x)=1$ for $x \in B_{r}$ and $|\nabla \varphi| \leq C / r$ using $\varphi^{p}$ as a test function in (6); we get

$$
\int_{B_{2 r}}-\operatorname{div}\left(|\nabla u|^{p-2} \nabla u\right) \varphi^{p} u+\int_{B_{2 r}} V|u|^{p-2} u \varphi^{p} u=0 .
$$

Thus,

$$
\begin{aligned}
\int_{B_{2 r}}|\nabla u|^{p} \varphi^{p} \\
\quad=-p \int_{B_{2 r}}|\nabla u|^{p-2} \varphi^{p-2} \nabla u \cdot \nabla \varphi(\varphi u)-\int_{B_{2 r}} V|\varphi u|^{p} .
\end{aligned}
$$

Using Young's inequalities for $(p-1) / p+1 / p=1$, we can estimate the first integral in the right-hand side of (47) by

$$
(p-1) \varepsilon^{p /(p-1)} \int_{B_{2 r}}|\nabla u|^{p} \varphi^{p}+\varepsilon^{-p} \int_{B_{2 r}}|\nabla \varphi|^{p}|u|^{p} .
$$

Also by result of Corollary 16, we can estimate the second integral in the right-hand side of (47) by

$$
\varepsilon \int_{B_{2 r}}|\nabla(\varphi u)|^{p}+C_{\varepsilon} \int_{B_{2 r}}|\varphi u|^{p} .
$$

Using these estimates in (47), we have

$$
\begin{aligned}
\int_{B_{2 r}} & |\nabla u|^{p} \varphi^{p} \\
\leq & \left((p-1) \varepsilon^{p /(p-1)}+\varepsilon\right) \int_{B_{2 r}}|\nabla u|^{p} \varphi^{p} \\
& +\left(\varepsilon^{p}+\varepsilon\right) \int_{B_{2 r}}|u|^{p}|\nabla u|^{p}+C_{\varepsilon} \int_{B_{2 r}}|\nabla u|^{p}|\varphi|^{p}
\end{aligned}
$$

Using the fact that $|\nabla \varphi| \leq C / r,|\varphi| \leq C / r$, and $\varphi=1$ in $B_{r}$, we immediately have inequality (45).

Lemma 18. Let $u \in C_{0}^{\infty}\left(B_{r}\right)$ where $B_{r}$ is the ball of radius $r$ in $\mathbb{R}^{n}$ and let $E=\left\{x \in B_{r}: u(x)=0\right\}$. Then, there exists $a$ constant $\beta$ depending only on $n$, such that

$$
\int_{A}|u| \leq \beta \frac{r^{n}}{m(E)}[m(A)]^{1 / n} \int_{B_{2 r}}|\nabla u|,
$$

for all ball $B_{r}$, $u$ as above, and all measurable sets $A \subset B_{r}$.

To prove this lemma see [5]. Note that $m(A)$ and $m(E)$ denote the Lebesgue measure of the sets $A$ and $E$.

\section{Strong Unique Continuation}

In this section, we proceed to establish the strong unique continuation property of the eigenfunction for the $p$-Laplacian operator in the case $V \in \widetilde{M}_{p}(\Omega)$.

Theorem 19. Let $u \in C_{0}^{\infty}(\Omega)$ be a solution of (6). If $u=0$ on a set $E$ of positive measures, then $u$ has zero of infinite order in p-mean.

Proof. We know that almost every point of $E$ is a point of density of $E$. Let $x_{0} \in E$ be such point. This means that

$$
\lim _{r \rightarrow 0} \frac{m\left(E \cap B_{r}\right)}{m\left(B_{r}\right)}=1,
$$

where $B_{r}$ denotes the ball of radius $r$ centered at $x_{0}$, and thus, given that $\varepsilon>0$, there is an $r_{0}=r_{0}(\varepsilon)$, such that

$$
\frac{m\left(E^{c} \cap B_{r}\right)}{m\left(B_{r}\right)}<\varepsilon, \quad \frac{m\left(E \cap B_{r}\right)}{m\left(B_{r}\right)}>1-\varepsilon \text { for } r \leq r_{0},
$$

where $E^{c}$ denotes the complement of the set $E$. Taking $r_{0}$, smaller if necessary, we can assume that $B_{r_{0}} \subset \Omega$. Since $u=0$ on $E$, by Lemmas 18 and 11, we have

$$
\begin{aligned}
& \int_{B_{r}}|u|^{p}= \int_{B_{r} \cap E^{c}}|u|^{p} \leq \beta \frac{r^{n}}{m\left(E \cap B_{r}\right)}\left[m\left(E^{c} \cap B_{r}\right)\right]^{1 / n} \\
& \times \int_{B_{r}}|\nabla(u)|^{p} \\
& p \beta \frac{r^{n} \varepsilon^{1 / n}}{\left[m\left(B_{r}\right)\right]^{1-1 / n}(1-\varepsilon)} \int_{B_{r}}|u|^{p-1}|\nabla(u)| .
\end{aligned}
$$

By Hölder inequality

$$
\int_{B_{r}}|u|^{p} \leq C \frac{\varepsilon^{1 / n}}{1-\varepsilon}\left(\int_{B_{r}}|\nabla(u)|^{p}\right)^{1 / p}\left(\int_{B_{r}}|u|^{p}\right)^{(p-1) / p},
$$

and by using the Young inequality, we get

$$
\int_{B_{r}}|u|^{p} \leq C \frac{\varepsilon^{1 / n}}{1-\varepsilon} r\left(r^{p-1} \int_{B_{r}}|\nabla(u)|^{p}+\frac{p-1}{r} \int_{B_{r}}\left|u^{p}\right|\right) .
$$

Finally, by Lemma 17, we have

$$
\int_{B_{r}}|u|^{p} \leq C \frac{\varepsilon^{1 / n}}{1-\varepsilon} \int_{B_{2 r}}\left|u^{p}\right|,
$$

where $C$ is independent of $\varepsilon$ and of $r$, as $r \rightarrow 0$. Now, let us introduce the following function:

$$
f(r)=\int_{B_{2 r}}|u|^{p}
$$

And let us fix $n \in \mathbb{N}$ and choose $\varepsilon>0$ such that $\left(C \varepsilon^{1 / n}\right)(1-$ $\varepsilon) \leq 2^{-n}$. Observe that consequently $r_{0}$ depends on $n$. Then, (57) can be written as

$$
f(r) \leq 2^{-n} f(2 r), \quad \text { for } r \leq r_{0} .
$$


Iterating (59), we get

$$
f(\rho) \leq 2^{-k n} f\left(2^{k} \rho\right), \quad \text { if } 2^{k-1} \rho \leq r_{0} .
$$

Now, given that $0<r<r_{0}(n)$, choose $k \in \mathbb{N}$, such that

$$
2^{-k} r_{0} \leq r \leq 2^{-k+1} r_{0} .
$$

From (60), we obtain

$$
f(r) \leq 2^{-k n} f\left(2^{k} r\right) \leq 2^{-k n} f(2 r) .
$$

Since $2^{-k} \leq r / r_{0}$, we finally obtain

$$
f(r) \leq\left(\frac{r}{r_{0}}\right)^{n} f\left(2 r_{0}\right) .
$$

And thus, we have

$$
\int_{B_{r}\left(x_{0}\right)}|u(y)|^{p} d y \leq\left(\frac{r}{r_{0}}\right)^{n} f\left(2 r_{0}\right)
$$

and this shows that (7) holds, which means that $u$ has a zero of infinite order in $p$-mean at $x_{0}$.

Corollary 20. Equation (6) has a strong unique continuation property.

\section{Acknowledgments}

The authors would like to thank the referee for the useful comments and suggestions which improved the presentation of this paper. The authors were supported by the Banco Central de Venezuela.

\section{References}

[1] M. Aizenman and B. Simon, "Brownian motion and harnack inequality for Schrödinger operators," Communications on Pure and Applied Mathematics, vol. 35, no. 2, pp. 209-273, 1982.

[2] P. Zamboni, "Unique continuation for non-negative solutions of quasilinear elliptic equations," Bulletin of the Australian Mathematical Society, vol. 64, no. 1, pp. 149-156, 2001.

[3] M. Schechter, Spectra of Partial Differential Operators, vol. 14, North-Holland, Amsterdam, The Netherlands, 2nd edition, 1986.

[4] C. L. Fefferman, "The uncertainty principle," Bulletin of the American Mathematical Society, vol. 9, no. 2, pp. 129-206, 1983.

[5] F. Chiarenza and M. Frasca, "A remark on a paper by C. Fefferman," Proceedings of the American Mathematical Society, vol. 108, no. 2, pp. 407-409, 1990.

[6] D. G. de Figueiredo and J.-P. Gossez, "Strict monotonicity of eigenvalues and unique continuation," Communications in Partial Differential Equations, vol. 17, no. 1-2, pp. 339-346, 1992.

[7] D. Jerison and C. E. Kening, "Unique continuation and absence of positive eigenvalues for Shrödinger operators: with an appendix by E. M. Stein," Annals of Mathematics, vol. 121, no. 3, pp. 463-494, 1985.

[8] N. Bourbaki, "Elements of Mathematics," in General Topology, Part 1, Addison Wesley.

[9] R. E. Castillo, "Nonlinear Bessel potentials and generalizations of the Kato class," Proyecciones, vol. 30, no. 3, pp. 285-294, 2011. 


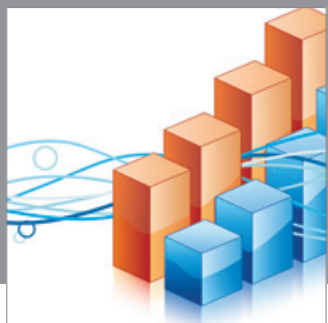

Advances in

Operations Research

mansans

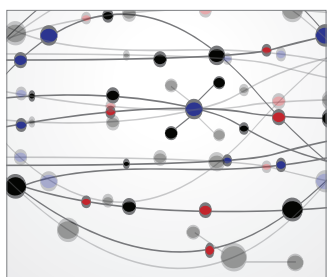

The Scientific World Journal
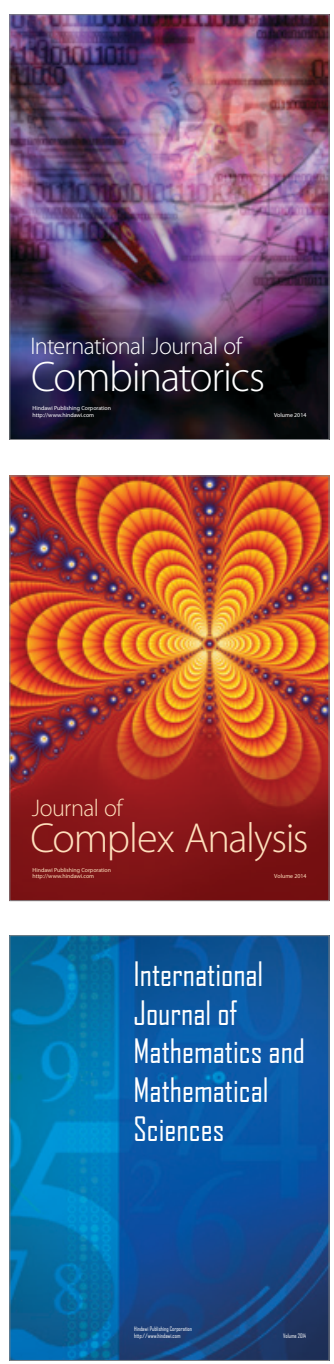
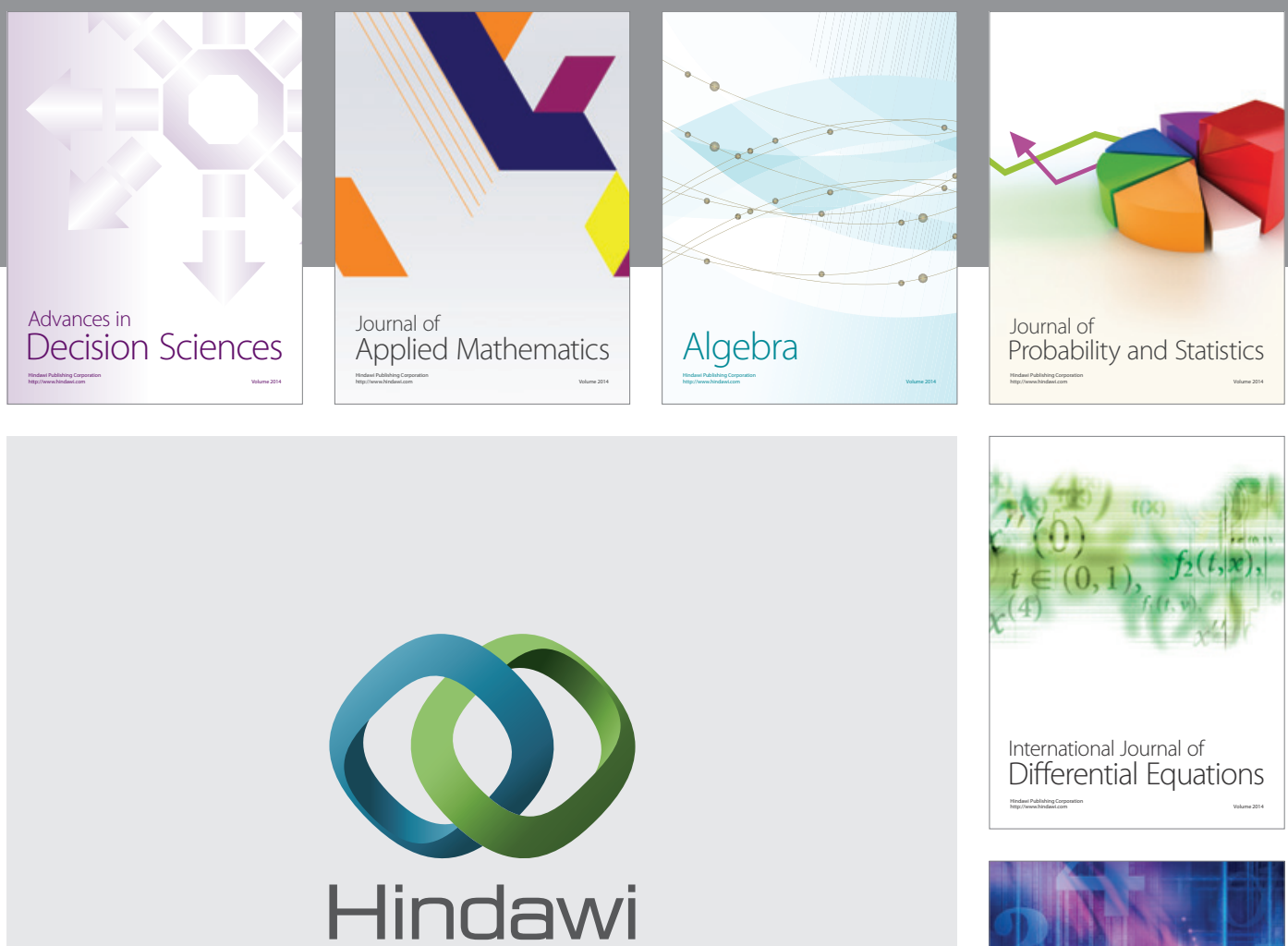

Submit your manuscripts at http://www.hindawi.com
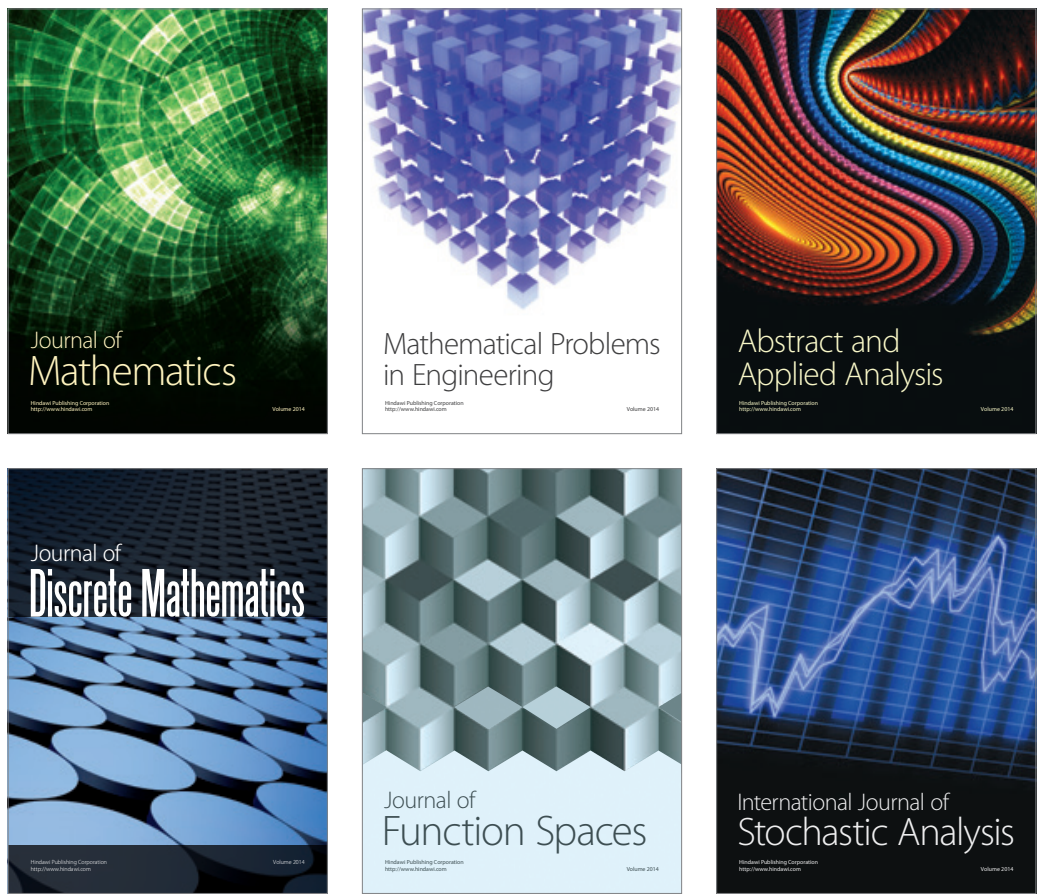

Journal of

Function Spaces

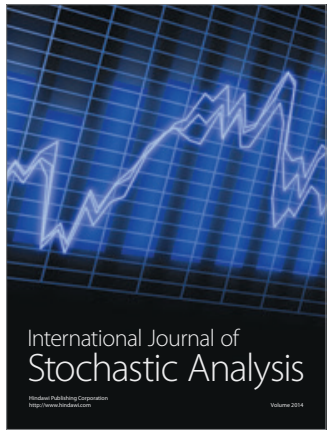

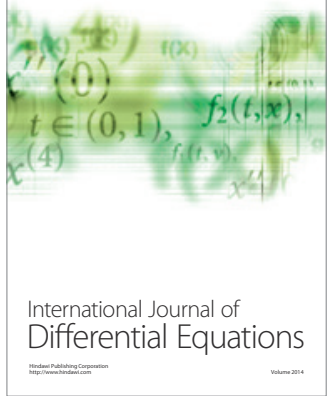
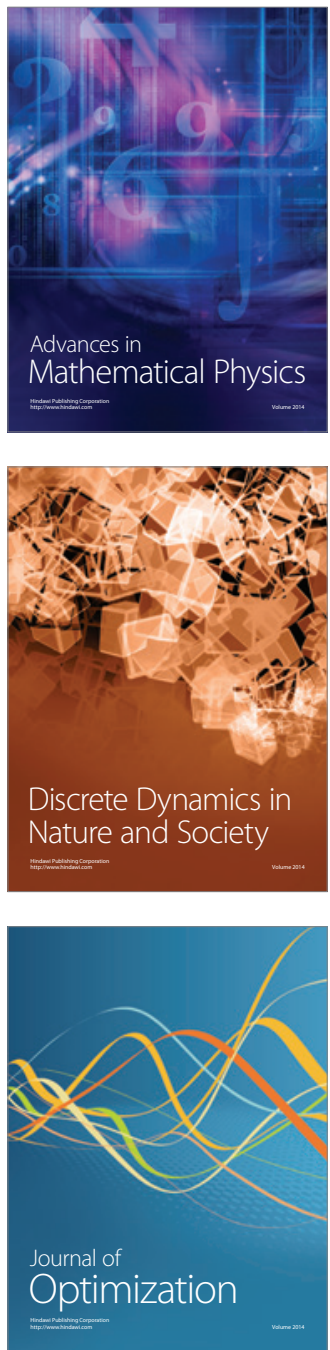